\title{
1 Aging increases the systemic molecular degree of inflammatory perturbation in patients
}

\section{2 with tuberculosis}

3 Deivide Oliveira-de-Souza1,2,3¥, Caian L. Vinhaes1,2,3z, María B. Arriaga1,2, Nathella Pavan

4 Kumar4, Artur T. L. Queiroz1,2, Kiyoshi F. Fukutani1,2,3, Subash Babu4,5, Bruno B.

5 Andrade $1,2,3,6,7,8^{*}$

6 1Instituto Gonçalo Moniz, Fundação Oswaldo Cruz, Salvador 40296-710, Brazil

7 2Multinational Organization Network Sponsoring Translational and Epidemiological Research

8 (MONSTER) Initiative, Salvador 41810-710, Brazil

9 3Curso de Medicina, Faculdade de Tecnologia e Ciências (FTC), Salvador 40290-150, Brazil

10 4National Institutes of Health- National Institute for Research in Tuberculosis, International

11 Center for Excellence in Research, Chennai, 600031, India.

12 5Laboratory of Parasitic Diseases, NIAID, NIH, Bethesda, 20892, USA

13 6Escola Bahiana de Medicina e Saúde Pública (EBMSP), Salvador, 40290-000, Brazil

147 Universidade Salvador (UNIFACS), Laureate Universities, Salvador, 41720-200, Brazil

15 sWellcome Centre for Infectious Diseases Research in Africa (CIDRI-Africa), Institute of

16 Infectious Disease and Molecular Medicine, University of Cape Town, Cape Town, South

17 Africa.

18 ¥These authors equally contributed to the work.

*Corresponding author: Bruno B. Andrade (bruno.andrade@fiocruz.br). 


\section{Abstract}

22 Tuberculosis (TB) in a chronic infection that can affect individuals of all ages. The description of 23 determinants of immunopathogenesis in TB is a field of tremendous interest due to the perspective 24 of finding a reliable host-directed therapy to reduce disease burden. The association between 25 specific biomarker profiles related to inflammation and the diverse clinical disease presentations 26 in TB has been extensively studied in adults. However, relatively scarce data on profiling the 27 inflammatory responses in pediatric TB are available. Here, we employed the molecular degree 28 of perturbation (MDP) score adapted to plasma biomarkers in two distinct databanks from studies 29 that examined either adults or children presenting with pulmonary or extrapulmonary disease. We 30 used multidimensional statistical analyses to characterize the impact of age on the overall changes 31 in the systemic inflammation profiles in subpopulation of TB patients. Our findings indicated that 32 TB results in significant increases in MDP values, with the highest values being detected in adult 33 patients. Furthermore, there were unique differences in the biomarker perturbation patterns and 34 the overall degree of inflammation according to disease site and age. Importantly, the molecular 35 degree of perturbation was not influenced by sex. Our results revealed that aging is an important 36 determinant of the differences in quality and magnitude of systemic inflammatory perturbation in 37 distinct clinical forms of TB.

38 Keywords: Tuberculosis; Biomarkers; Inflammation; molecular degree of perturbation; Mycobacterium tuberculosis. 


\section{Introduction}

42 Tuberculosis (TB) remains the leading cause of mortality worldwide due to a single agent [1].

43 Mycobacterium Tuberculosis (Mtb) is widely disseminated geographically and infects individuals

44 of all ages, causing a wide spectrum of clinical manifestations associated with the host 45 immunological status [2].

46 The majority of the studies exploring immunopathogenesis in TB is restricted to the adult 47 population. On the other hand, TB pathophysiology remains poorly understood in children, 48 especially in those under 5 years-old [3-5]. An important challenge in pediatric TB is the increased 49 frequency of extrapulmonary presentations [6], which can be paucibacillary and thus associated 50 with challenges in microbiologic test confirmation, resulting in delayed therapy implementation.

51 Inflammatory biomarkers in TB have been extensively studied and gained prominence due to the potential use as host-based blood tests [7]. Understanding the complexity of inflammatory milieu after TB infection in children is the key to expand the field and start development of new tools to

54 aid the clinical management and contribute to non-sputum-based point of care test, with progress 55 in diagnosis and clinical management.

56 Here, we used an adaptation of the molecular degree of perturbation (MDP), as previously published by us $[8,9]$ to assess the nuances of TB in pediatric patients compared to those observed in adult patient population. We found that the degree of inflammatory imbalance is associated with aging, suggesting that TB patients develop augmented capacity of promoting systemic inflammation with increasing age. Given that TB clinical presentation is a result of immunopathology, our results reinforce the idea that the distinct inflammatory profile in blood underlies the differences observed in TB disease presentation between adults and children. 


\section{Ethics statement}

All clinical investigations were conducted according to the principles expressed in the Declaration of Helsinki. Written informed consent was obtained from all participants or their legally responsible guardians before enrolling into the sub-studies. The study was approved by the Institutional Review Board of the National Institute for Research in Tuberculosis, Chennai, India (NIRT; protocol numbers NCT01154959 and NCT00342017).

\section{Study design and participants}

Active TB cases were recruited at the Government Stanley Medical Hospital, at TB clinics supported by the National Institute for Research in Tuberculosis and Childs Trust Hospital in Chennai, India. Detailed information on diagnosis of PTB in adults have been described previously in $[8,10]$ whereas the procedures used for diagnosis of PTB and EPTB in children have been reported in $[11,12]$. Briefly, the diagnosis of PTB in adults and children was based on sputum smear and culture positivity. EPTB in adults was diagnosed on the basis of AFB staining and/or culture positivity of fine-needle aspiration biopsies of lymph nodes or pleural effusions in adult. The diagnosis of EPTB in children was made on clinical symptoms, physical examination and biopsies according to the site of clinical manifestation, such as fine-needle aspiration for the cases of TB lymphadenitis or cerebrospinal fluid analysis for TB meningitis as described in [12]. At the time of enrollment, all active TB cases had no record of prior TB disease or anti-TB treatment (ATT). The healthy control adults were asymptomatic with normal chest X-rays, negative TST (indurations $<5 \mathrm{~mm}$ in diameter) and QuantiFERON TB Gold-in-Tube enzymelinked immunosorbent assay (Cellestis), as well as negative sputum smear or culture results as described in $[8,10]$. Pediatric participants included in the heathy control group were asymptomatic who went to the hospital for routine vaccinations and tested negative in the QuantiFERON TB assay. All participants were BCG vaccinated and were HIV negative. Plasma samples were collected from a total of 152 adults and 54 children. Among adults, 97 were diagnosis of PTB, 35 of EPTB and 20 were healthy controls. Within children, 14 had PTB, 22 had EPTB and 18 were healthy controls. Adults with EPTB had TB lymphadenitis $(n=24)$ or pleural TB $(n=11)$ whereas children presented with spinal TB $(n=14)$, TB lymphadenitis $(n=6)$, and abdominal TB $(n=2)$, which included peritonitis or tuberculomas. All participants were recruited in Chennai, India, as part of a large TB natural history study. 


\section{Immunoassays}

We evaluated a panel of 17 cytokines, tissue remodeling mediators and matrix metalloproteinases to examine molecular degree of inflammatory perturbation using different immunoassays. In the original study, biomarkers were measured in EDTA-treated plasma samples. Biomarkers included were cytokines, acute-phase proteins and tissue remodeling proteins. Bio-Plex multiplex ELISA cytokine assay system (R\&D Systems) was employed to measure the cytokines analyzed. The list of cytokines included interleukin (IL)-1 $\beta$, IL-10, IL-12p70, IL-17, interferon (IFN)- $\gamma$ and tumor necrosis factor $\alpha$ (TNF- $\alpha$ ). Moreover, IFN- $\alpha$ and IFN- $\beta$ levels were quantified using the VeriKine serum ELISA kit (PBL Interferon Source). Plasma levels of vascular endothelial growth factor (VEGF) was measured using the Milliplex map kit system by Merck Millipore. Concentration of extracellular matrix metalloproteinases (MMPs)-1, 8 and 9, and tissue inhibitors of metalloproteinases (TIMPs)-1, 2, 3 and 4 were measured using Luminex technology (R\&D Systems), according to the manufacturer's protocols. Plasmatic Hemoxygenase 1 (HO-1) was measured by ELISA (Assay Designs).

\section{Data Analysis}

Categorical data were presented as proportions and continuous data as medians and interquartile ranges (IQR). The Fisher's exact test was used to compare categorical variables between study groups. Continuous variables were compared using the Mann-Whitney $U$ test. P-values were adjusted for multiple measurements using Holm-Bonferroni's method. Hierarchical cluster analyses (Ward's method) of z-score normalized data were employed to depict the overall expression profile of indicated biomarkers in the study groups. Dendrograms represent Euclidean distance.

Profiles of correlations between biomarkers in different clinical groups were examined using network analysis of the Spearman correlation matrices (with 100X bootstrap). In indicated analyses, only correlations with significant adjusted P-value by the number of measurements (established cut-off was P-value <0.003) were included in the network visualization. In such analyses, markers that exhibited similar correlation profiles were clustered based on a modularity [13], which infers a sub-networks inside the of the correlation network profiles and depicted using Fruchterman Reingold (force-directed graph drawing)[14].

Sparse canonical correlation analysis (CCA) modeling was employed to assess whether combinations of circulating biomarkers could discriminate between subgroups of patients. The CCA model was chosen because many variables were studied. This model is able to perform dimensionality reduction for two co-dependent data sets (MDP biomarker profile and baseline characteristics profile, which were age and sex) simultaneously so that the discrimination of the 
clinical endpoints represents a combination of variables that are maximally correlated. Thus, trends of correlations between parameters in different clinical groups rather than their respective distribution within each group are the key components driving the discrimination outcome. In our CCA model, simplified and adapted from previously reported investigations of biomarkers for TB diagnosis [8, 15-18]. In the biomarker profile dataset, we included values of all the inflammatory marker variables. The diagnostic class prediction values obtained were calculated using receiver operator characteristics curve analysis. Probability of being molecularly perturbed according to increases in age was calculated using Kaplan-Meyer curves.

\section{Adaptation of the Molecular Degree of Perturbation to examine plasma concentrations of biomarkers}

The plasma measurements of both datasets were normalized equally with a $\log 2$ transformation and the batch effect within the different study datasets was corrected using Combat algorithm from SVA package [16]. The ComBat algorithm is a widely used method for adjusting batch effects in microarray and RNA-Seq data associated with technical variance effects. The molecular inflammatory perturbation is based molecular degree of perturbation (MDP) method used in the present study is an adaptation of the MDH described by Pankla et al. [19]. In the present study, instead of using gene expression values as in Prada-Medina et al. [20] we inputted plasma concentrations of a defined set of biomarkers pre-selected based on previously published studies from our group which investigated TB pathogenesis $[15,21]$. Thus, herein, the average plasma concentration levels and standard deviation of a baseline reference group (healthy uninfected controls) were calculated for each biomarker. The MDP score of an individual biomarker was defined by the differences in concentration levels from the average of the biomarker in reference group divided by the reference standard deviation. Essentially, the MDP score represents the differences by number of standard deviations from the healthy control group. The formulas used to calculate MDP in the present study are shown below:

$$
\text { Molecular degree of perturbation }=\frac{\mathrm{x}_{\mathrm{i}}-\overline{\mathrm{x}}_{(\text {reference })}}{\sigma_{(\text {reference })}}
$$

$\mathrm{n}=$ Number of data points

$\mathrm{Xi}=$ Each of the value of data

$$
\sigma=\frac{\sum_{\mathrm{i}=1}^{\mathrm{n}}\left(\mathrm{x}_{\mathrm{i}}-\overline{\mathrm{x}}\right)^{2}}{\mathrm{n}-1}
$$

$\overline{\mathrm{x}}=$ Mean of the data points

$\sigma=$ Standard deviation

In this study, we applied the MDP scoring system using data on 17 biomarkers measured from two distinct groups of patients, adults and children with active TB and healthy uninfected controls. 
164 The MDP transformation was used as an approach to normalize data cross experiments resulting

165 in datasets with markers distributed in a similar scale.

166 The MDP was filtered by the absolute MDP scores below 2 module and sum of all deviations

167 accumulated MDP. To identify which sample was "perturbed", we calculated the cutoff of the

168 average MDP scores plus 2 standard deviations of the reference group and all values above this

169 threshold was considered "perturbed".

170 


\section{$171 \quad$ Results}

\section{Characteristics of study participants}

173 Baseline characteristics of the adult and pediatric participants have been described elsewhere [12].

174 Adults with pulmonary TB and uninfected healthy controls were more frequently males than those 175 with extrapulmonary TB $(65.9 \%$ vs. $85 \%$ vs. $45.7 \%$, respectively; $\mathrm{P}=0.006)$. In addition, adults 176 with PTB were on average older than healthy controls (median [IQR] in years: 38 [28-47] vs. 28.5 177 [26-35], respectively; $\mathrm{P}=0.005$ ) but had median age similar to the group of EPTB (Table S1). In 178 the pediatric study, patients with PTB were similar to those presenting with EPTB or HC with 179 regard age (median [IQR] in years: 6.5 [1.7-12.5] vs. 7 [3.7-13] vs. 10 [3-12], respectively; $\mathrm{P}=$ 180 0.684) and sex $(\mathrm{P}=0.393)$, with and overall high frequency of male individuals $(42.8 \%$ vs. 59.1\% 181 vs. 66.7\%) (Table S2). We next compared the adults and children and found that there were no 182 significant differences in sex distribution in comparisons between the $\mathrm{HC}$, the PTB as well as the 183 EPTB subgroups (Table S3). 

mediators in adult and children with active tuberculosis

Plasma levels of 17 cytokines and tissue remodeling mediators were compared between pulmonary tuberculosis (PTB) and extrapulmonary tuberculosis (EPTB) and uninfected healthy controls (HC) in adult and children from India, separately (concentration values are described in Table S4 and S5). In adults, compared to HC, the PTB or EPTB groups exhibited higher levels of most parameters, except for IFN- $\beta$, TIMP-2, IL-12p70 and TIMP-4 which were not statistically different (Table S4). In the pediatric population, individuals with active TB (PTB or EPTB) exhibited on average higher levels of fewer markers (HO-1, MMP-1, MMP-8, TIMP-1 and TIMP3) than controls (Table S5). These results suggested that TB in adults may lead to more significant changes in the concentration levels of plasma biomarkers than what we observed in children with this condition. directly compare the groups from the distinct studies, we calculated the overall MDP score values according to our previous publication [8], that active TB was associated with a substantial increase in MDP scores compared to HC in both adults (PTB $p<0.0001$, EPTB $p<0.0001$; Figure 1A) and children (PTB p <0.001, EPTB p=0.002; Figure 1B). Adult patients with PTB exhibited higher MDP values than those with EPTB ( $p=0.0007$; Figure 1A), whereas MDP values in PTB and EPTB were indistinguishable in children ( $p>0.999$; Figure 1B). Interestingly, adult patients with either PTB or EPTB exhibited higher MDP values than those from the pediatric population (adults PTB vs. children PTB: $p<0.0001$; adults EPTB vs. children EPTB: $p<0.001$; Figure 1C) suggesting that the impact of Mtb on changes in the systemic molecular degree of perturbation is likely influenced by age. Reinforcing this idea, we found no differences in MDP values between 
210 Plasma markers driving the overall molecular degree of perturbation in tuberculosis are

\section{1 distinct between adults and children}

212 We examined the MDP expression values for each individual plasma cytokine and tissue remodeling mediators. An unsupervised hierarchical clustering was used to test whether the overall expression profile was associated with specific changes in active TB (PTB or EPTB) and

$215 \mathrm{HC}$ in adults and children. We found that overall expression profile was very distinct between adults with active tuberculosis and HC. (Figure $\mathbf{2 A}$, left panel). Intriguingly, children showed heterogenous profile expression of the 17 markers between active TB and HC (Figure 2B, left panel), without a clear separation in the cluster analysis. These findings indicate that the overall expression profile of the MDP values can be used to distinguish active TB from controls in adults but not in children. In addition, PTB could not be grouped separately from EPTB in both adults and children (Figure 2A and Figure 2B, left panels), indicating that active TB drives specific changes in MDP independent on disease site. Univariate analyses comparing the MDP values for each marker between HC and PTB or EPTB groups were showed in Figure S1and Figure S2. from all markers to identify which one contributes the most for the discrimination between PTB or EPTB and $\mathrm{HC}$ in both adults and children. Curiously, the markers with the strongest contributions for discrimination in adults with PTB vs. HC were HO-1, IFN- $\gamma$, IL-17, IL-1 $\beta$ and TIMP-2 whereas in children such markers were TIMP-3, HO-1, VEGF, TIMP-4 and MMP-9 (Figure 2A-B right panels). The canonical model also indicated the markers that contributed the most for the discrimination EPTB vs. HC in adults were IL- $1 \beta$, IFN- $\gamma$, IFN- $\alpha$, MMP-8 and HO1, whereas in children were IL-17, HO-1, MMP-1, IL-1 $\beta$ and TNF- $\alpha$ (Figure 2A-B right panels). Of note, the MDP values of 3 out of 5 markers which were relevant to identify PTB in adults (HO-1, IFN- $\gamma$ and IL-1 $\beta$ ) were also part of TB signature observed in adults with EPTB. Moreover, in children, only 1 marker which was relevant to identify PTB (HO-1) was also relevant in EPTB. These findings indicate that in the context of Mtb infection, the plasma markers likely to be differentially perturbed according to disease site and age, except for HO-1. 

between adults and children.

To understand the nuances between molecular degree of perturbation of individual markers and their direct effect on overall MDP values, we employed network analysis based on Spearman correlation matrices, as previously described [8]. Using this approach, we found that the presence of pulmonary infection in adults was associated a greater number of correlations when compared with those that developed EPTB (Figure 3A). The group of adults with PTB was marked by several positive correlations, highlighting that the degree of perturbation in HO-1, IFN- $\alpha$, IFN- $\gamma$, IL-10, IL-17, IL-1 $\beta$ and TIMP-1 markers was directly associated with the overall MDP (Figure 3A, left panel). In addition, perturbation of MMP-1 was inversely correlated with the overall MDP values in this clinical group. Furthermore, the top nodes exhibiting the highest number of significant correlations in the network of adults with PTB were MDP followed by MMP-1, IL10, HO-1 and MMP-8. Importantly, the correlation profile found in the adult with EPTB was distinct from PTB (Figure 3A). Indeed, only the degree of perturbation of HO-1 and IFN- $\gamma$ were directly associated with the overall MDP values in the EPTB group. Node analysis of EPTB network demonstrated that MMP-8, IFN- $\gamma$, overall MDP and HO-1 and were the most highly connected parameters (Figure 3A, right panel).

When the network analysis was extended to the pediatric population, we observed a decreased number of statistically significant correlations in pulmonary infection when compared with extrapulmonary TB (Figure 3B). Interestingly, we found that Mtb infection was associated with marked absence of negative correlations in children. Node analysis of the PTB network indicated that IL-12p70, IL-1 $\beta$ and TNF- $\alpha$ were the most highly connected markers (Figure 3B, left panel). Curiously, the overall MDP values, which were highly connected in the networks from adults, were statistically correlated only with perturbation of HO-1 and TIMP-1. Children with extrapulmonary TB had TNF- $\alpha$, followed by IFN- $\alpha$, TIMP-3 and TIMP-1 as the most relevant nodes (Figure 3B, right panel). The degree of perturbation of HO-1, IFN- $\alpha$, TIMP-1 and TIMP-3 was directly associated with the overall MDP values in children with EPTB. Furthermore, in children, there was a lack of correlations between the MDP values of markers described to be important in TB pathogenesis such as IFN- $\gamma$, IL-1 $\beta$ and TNF- $\alpha$ in the networks. molecular perturbation in active TB in adults and children. 

independent of sex

272 The results described above suggested that age was associated with the molecular degree of 273 inflammatory perturbation. To directly test this hypothesis, we grouped each individual based on 274 age and performed an exploratory investigation using unsupervised hierarchical cluster analysis 275 with z-score normalized values of the MDP calculated for marker. We found that PTB did not 276 exhibit a distinct biomarker MDP profile compared to EPTB independent on age (Figure 4A, left 277 panel). Of note, MDP values detected for many markers were relatively lower in children 278 independent on the TB clinical presentation, except for TIMP-1, TIMP-2, TIMP-3 and TIMP-4, which tended to be higher in those who were younger (Figure 4A, left panel). We next tested direct correlations between age and the individual MDP values of each marker. Spearman correlation analysis revealed that the degree of perturbation of IFN- $\gamma$, IL-1 $\beta$, TNF- $\alpha$, IFN- $\alpha$, HO1, IL-17, MMP-1, MMP-8 and VEGF was directly correlated whereas TIMP-2 values were inversely correlated with age (Figure 4A, right panel).

To determine association between age and probability of being molecularly perturbed (see Methods for definition) in the entire population, we used a model adapted from the KaplanMeier survival curve (Figure 4B). This approach revealed that increase in age was directly associated with higher probability of overall molecular perturbation. Indeed, the overall MDP score values were positive correlated with age in all the clinical groups evaluated (Figure S3). Finally, we examined the influence of sex on the association between age and inflammatory perturbation. Overall MDP values were not different between male and female individuals stratified in the distinct clinical groups (Figure 5A). Furthermore, using the Kaplan-Meier survival curve test, we found that, in general, there was no different in the curves of female and male participants $(\mathrm{p}=0.29$, Figure 5A). Spearman correlation analysis demonstrated that MDP values of most of markers were positively correlated with age in both male and female participants (Figure 5B). TIMP-2 was the only markers with a negative correlation in females. These findings suggested that sex does not influence the higher probability of inflammatory perturbation with aging in TB. 
Mechanisms of disease pathogenesis in TB have been extensively studied over the years, but despite that, the immunopathology of this infection in pediatric populations remains poorly understood. Importantly, Mtb infection is one of the main causes of childhood morbidity and mortality worldwide [11, 22, 23], and the field needs elucidation of the determinants of immunopathogenesis. In the present study, we used an adaption of molecular degree of perturbation $[8,9]$ to estimate the level and quality of systemic inflammation in patients with active TB (PTB and EPTB) according to age. Our findings indicated that there are important discrepancies in the MDP values between adults and children with active TB, with adults exhibiting higher values, whereas the molecular perturbation was similar among individuals from the healthy control groups independent of age. Thus, while the systemic inflammatory profile is similar between adults and children without TB, it becomes very distinct in patients with active disease. Although we have not directly tested potential influence of maturation of immune system in the results, it is possible that the capacity to promote significant systemic inflammation in the context of TB may be affected by this process.

Mycobacterial infection is known to cause profound stimulation of both innate and adaptive immune response, in vitro and in vivo models $[24,25]$. Our exploratory analysis characterized the systemic inflammatory response and indicated that Mtb infection (pulmonary and extrapulmonary) was associated with overall increases in the MDP values in both adults and children. However, in either PTB or EPTB groups, adult patients exhibited augmented MDP values compared to pediatric patients. This finding reinforces the idea that in the context of TB, adults are more prone towards presenting with a higher degree of inflammation than children. We expanded these analyses to show the degree of perturbation of each individual biomarker and found that the in adults, individuals with active TB exhibit a very distinct profile compared to those without. Nevertheless, in the pediatric population, there was no clear combined biomarker profile that could distinguish TB from controls. Hence, there are specific changes in the biomarker MDP profile that are age dependent. Of note, using discriminant analyses based on a canonical model, we identified that the top markers responsible for the discrimination between the clinical groups differed between adults and children. In adults, HO-1, IFN- $\gamma$ and IL-1 $\beta$, markers which have been associated with TB pathogenesis [26-28] were the top markers that contributed to discrimination between the disease groups (both PTB and EPTB) and controls $[15,26,29]$ whereas in children, TIMP-3, IL-17 and HO-1 were the markers that most contributed for discrimination between active TB patients and uninfected controls. Of note, important finding of this analytical approach was that the molecular perturbation of HO-1 could discriminate pulmonary and extrapulmonary TB from healthy controls in both adult and children populations. HO-1 is 
critical role in cytoprotection [31]. These observations made us hypothesize HO-1 may be important in TB pathogenesis regardless of age. Additional studies in more diverse populations are warranted to test this idea.

The inflammatory process results from an intricate relationship between factors from the host and pathogen, and can be evaluated using network analysis [9, 32, 33]. Using this approach, we showed important differences in the correlation profiles between MDP values from each individual biomarker and the overall MDP values in adults and children with active TB. Pulmonary infection with Mtb in adults led to a coordinated inflammatory burst, which was read by detection of many statistically significant correlations between individual markers and overall MDP values. On the converse, in children presenting with PTB the augmented inflammation seemed less coordinated, meaning increases in molecular perturbation of a given marker were not followed by simultaneous increases of other markers or the overall MDP values. This suggests that pediatric PTB patients may have a decreased ability to mount a coordinated response. It is possible to hypothesize that such uncoupling of inflammatory activity may be a consequence of immune immaturity that leads to dissemination of bacilli in children, as previously showed [6]. Curiously, in children with EPTB, the networks were more complex, indicating a higher number of connections. Such phenomenon could be consequence of extrapulmonary tissue damage, and maybe argue that despite the lower capacity to mount and sustain a coordinated response in pulmonary infection, EPTB in children is marked by a more balanced interplay between innate and adaptive immune response [24]. Moreover, in adults with EPTB, the complexity of the inflammatory network was reduced, in agreement with the previous published evidence of probably less organized and an unfettered immune activation in adults with EPTB [9]. The determinants of the differences in correlations between concentrations of mediators of inflammation between distinct clinical forms of TB in adults and children are still unknown.

An interesting result reported here was the association between increases in age and augmentation of the overall molecular degree of perturbation in both PTB and EPTB patients. Indeed, our results demonstrated that increase in age leads to rise in probability of a TB patient becoming more perturbed, independent on disease site (PTB or EPTB). Importantly, we showed that degree of perturbation of many individual plasma markers correlated with age, reinforcing the idea that the capacity to induce systemic inflammation is proportional to age. Furthermore, our analysis showed that sex did not influence the probability of increase in MDP values, arguing that the potential effect of sex-related hormones on systemic inflammation may be superposed by the effect of aging in TB patients.

Our study has limitations. We were unable to test association between MDP and bacillary loads due to lack of data from the pediatric population. It is possible that higher MDP values 
370 detected in adults may be a consequence of the increased mycobacterial infection loads. In

371 addition, the sample size of the group of children was relatively small. Regardless, the extensive

372 exploratory analyses performed revealed unique relationships between age and the systemic

373 degree of inflammation. The results presented here shed light into the impact of aging on the

374 systemic immune activation during TB.

375 


\section{References}

377 1. Organization WH. Global Tuberculosis Report. 2019.

378 2. Pai M, Behr MA, Dowdy D, et al. Tuberculosis. Nat Rev Dis Primers 2016; 2:16076.

379 3. Starke JR. Resurgence of tuberculosis in children. Pediatr Pulmonol Suppl 1995; 11:16-7.

380 4. Schepisi MS, Motta I, Dore S, Costa C, Sotgiu G, Girardi E. Tuberculosis transmission among 381 children and adolescents in schools and other congregate settings: a systematic review. New 382 Microbiol 2019; 41:282-90.

383 5. Organization WH. A Research Agenda for Childhood Tuberculosis. 2007.

384 6. Li J, Sun L, Xu F, et al. Characterization of plasma proteins in children of different 385 Mycobacterium tuberculosis infection status using label-free quantitative proteomics. 386 Oncotarget 2017; 8:103290-301.

387 7. Silveira-Mattos PS, Barreto-Duarte B, Vasconcelos B, et al. Differential expression of activation 388 markers by Mycobacterium tuberculosis-specific CD4+ T-cell distinguishes extrapulmonary from 389 pulmonary tuberculosis and latent infection. Clin Infect Dis 2019.

390 8. Oliveira-de-Souza D, Vinhaes CL, Arriaga MB, et al. Molecular degree of perturbation of 391 plasma inflammatory markers associated with tuberculosis reveals distinct disease profiles between Indian and Chinese populations. Sci Rep 2019; 9:8002.

9. Vinhaes CL, Oliveira-de-Souza D, Silveira-Mattos PS, et al. Changes in inflammatory protein and lipid mediator profiles persist after antitubercular treatment of pulmonary and extrapulmonary tuberculosis: A prospective cohort study. Cytokine 2019; 123:154759.

10. Andrade BB, Pavan Kumar N, Amaral EP, et al. Heme Oxygenase-1 Regulation of Matrix Metalloproteinase-1 Expression Underlies Distinct Disease Profiles in Tuberculosis. J Immunol 2015; 195:2763-73.

11. Albuquerque VVS, Kumar NP, Fukutani KF, et al. Plasma levels of C-reactive protein, matrix metalloproteinase-7 and lipopolysaccharide-binding protein distinguish active pulmonary or extrapulmonary tuberculosis from uninfected controls in children. Cytokine 2019; 123:154773. 12. Pavan Kumar N, Anuradha R, Andrade BB, et al. Circulating biomarkers of pulmonary and extrapulmonary tuberculosis in children. Clin Vaccine Immunol 2013; 20:704-11.

13. Blondel VD, Guillaueme, J., Lamblotte, R. \& Lefebvre, E. Fast unfolding of communities in large networks. Journal of Statistical Mechanics: Theory and Experiment 2008.

14. S. BMH, M. J. In International AAAI Conference on Weblogs and Social Media, 2009.

15. Mayer-Barber KD, Andrade BB, Oland SD, et al. Host-directed therapy of tuberculosis based on interleukin-1 and type I interferon crosstalk. Nature 2014; 511:99-103.

16. Johnson WE, Li C, Rabinovic A. Adjusting batch effects in microarray expression data using empirical Bayes methods. Biostatistics 2007; 8:118-27.

411 17. Manabe YC, Andrade BB, Gupte N, et al. A Parsimonious Host Inflammatory Biomarker 412 Signature Predicts Incident TB and Mortality in Advanced HIV. Clin Infect Dis 2019.

413 18. Rousu J, Agranoff DD, Sodeinde O, Shawe-Taylor J, Fernandez-Reyes D. Biomarker discovery 414 by sparse canonical correlation analysis of complex clinical phenotypes of tuberculosis and malaria. PLoS Comput Biol 2013; 9:e1003018.

19. Pankla R, Buddhisa S, Berry M, et al. Genomic transcriptional profiling identifies a candidate blood biomarker signature for the diagnosis of septicemic melioidosis. Genome Biol 2009; 10:R127.

20. Prada-Medina CA, Fukutani KF, Pavan Kumar N, et al. Systems Immunology of DiabetesTuberculosis Comorbidity Reveals Signatures of Disease Complications. Sci Rep 2017; 7:1999.

21. Amaral EP, Ribeiro SC, Lanes VR, et al. Pulmonary infection with hypervirulent Mycobacteria reveals a crucial role for the P2X7 receptor in aggressive forms of tuberculosis. PLoS Pathog 2014; 10:e1004188.

22. Martinez L, Zar HJ. Tuberculin conversion and tuberculosis disease in infants and young children from the Drakenstein Child Health Study: A call to action. S Afr Med J 2018; 108:247-8. 
426 23. Sigal GB, Segal MR, Mathew A, et al. Biomarkers of Tuberculosis Severity and Treatment 427 Effect: A Directed Screen of 70 Host Markers in a Randomized Clinical Trial. EBioMedicine 2017; $428 \quad 25: 112-21$.

429 24. Scriba TJ, Coussens AK, Fletcher HA. Human Immunology of Tuberculosis. Microbiol Spectr $430 \quad 2016 ; 4$.

431 25. Cliff JM, Kaufmann SH, McShane H, van Helden P, O'Garra A. The human immune response to tuberculosis and its treatment: a view from the blood. Immunol Rev 2015; 264:88-102.

433 26. Mayer-Barber KD, Sher A. Cytokine and lipid mediator networks in tuberculosis. Immunol 434 Rev 2015; 264:264-75.

435 27. Mayer-Barber KD, Yan B. Clash of the Cytokine Titans: counter-regulation of interleukin-1 and type I interferon-mediated inflammatory responses. Cell Mol Immunol 2017; 14:22-35. 28. Novikov A, Cardone M, Thompson R, et al. Mycobacterium tuberculosis triggers host type I IFN signaling to regulate IL-1beta production in human macrophages. J Immunol 2011; 187:2540-7.

440 29. Mayer-Barber KD, Andrade BB, Barber DL, et al. Innate and adaptive interferons suppress IL1alpha and IL-1beta production by distinct pulmonary myeloid subsets during Mycobacterium tuberculosis infection. Immunity 2011; 35:1023-34. 30. Fredenburgh LE, Perrella MA, Mitsialis SA. The role of heme oxygenase- 1 in pulmonary disease. Am J Respir Cell Mol Biol 2007; 36:158-65. 31. Origassa CS, Camara NO. Cytoprotective role of heme oxygenase-1 and heme degradation derived end products in liver injury. World J Hepatol 2013; 5:541-9. 32. Andrade BB, Singh A, Narendran G, et al. Mycobacterial antigen driven activation of CD14++CD16- monocytes is a predictor of tuberculosis-associated immune reconstitution inflammatory syndrome. PLoS Pathog 2014; 10:e1004433.

450 33. Hsu DC, Breglio KF, Pei L, et al. Emergence of Polyfunctional Cytotoxic CD4+ T Cells in 451 Mycobacterium avium Immune Reconstitution Inflammatory Syndrome in Human Immunodeficiency Virus-Infected Patients. Clin Infect Dis 2018; 67:437-46. 
455 Acknowledgments: The authors acknowledge study participants. This project was supported by the Intramural Research Program of the NIAID to S.B. and N.P.K. This study was also financed in part by Coordenação de Aperfeiçoamento de Pessoal de Nível Superior (CAPES) (Finance Code 001). The work of B.B.A. was supported by grants from the NIH (U01AI115940, R01AI069923-08, R01AI20790-02), by Intramural Program of Fundação Oswaldo Cruz and by the Brazilian National Council for Scientific and Technological Development (CNPq). D.O.S. and M.B.A. receive fellowships from the Fundação de Amparo à Pesquisa da Bahia (FAPESB). C.L.V. is a research fellow and K.F.F. is a postdoctoral fellow from CNPq.

Author contributions: B.B.A. designed the study and mentored the work; D.O.S., C.L.V., M.B.A., N.P.K. performed the experiments and data collection; B.B.A., K.F.F., A.T.L.Q., M.B.A, D.O.S., C.L.V. performed data analyses; S.B. coordinated the clinical study, provided reagents for the immunoassays and helped with data interpretation; B.B.A., D.O.S., C.L.V., S.B. wrote the manuscript. All authors have read and approved the final version of the manuscript.

Data availability statement: The datasets generated during and/or analyzed during the current study are available from the corresponding author on reasonable request.

470 Competing interests: The authors declare that they have no financial of non-financial conflicts of interest. 
A

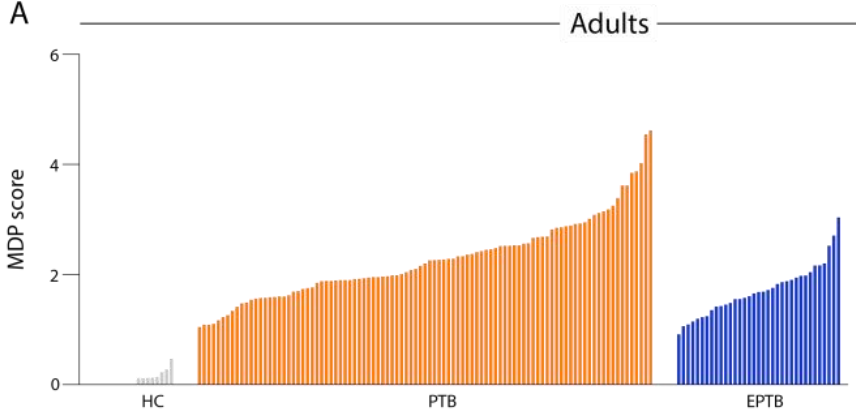

B$$
\text { ڤั̀ }
$$

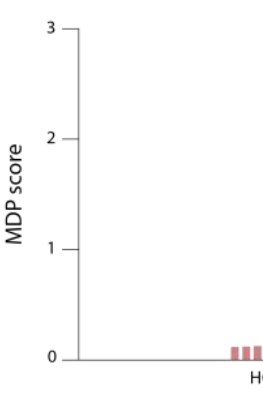

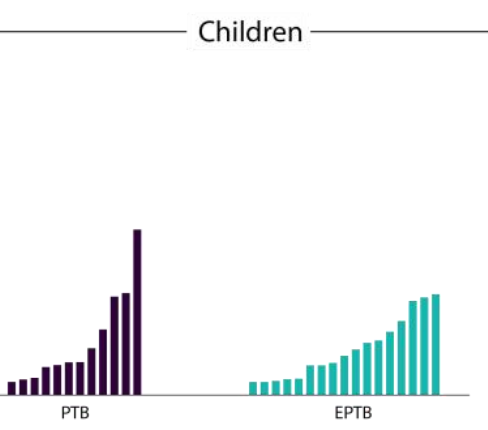

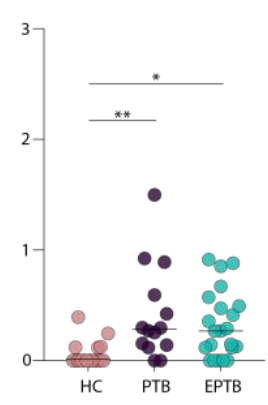

C

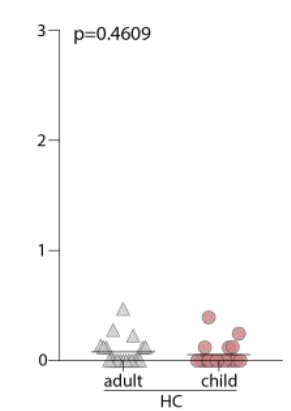

Figure 1: Adult and children with active tuberculosis exhibit substantial molecular degree of perturbation. $(\mathbf{A}, \mathbf{B})$ Left panels: Histograms show the single sample molecular degree of perturbation (MDP) score values relative to the healthy control group between adult and child (pulmonary TB: PTB, extrapulmonary TB: EPTB, healthy controls: HC). MDP values were calculated as described in Methods. The Kruskal Wallis test with Dunn's multiple comparisons was used to compare MDP values between each clinical group. Right panels: Scatter plots of the summary data for each group are shown. MDP score values were compared between PTB or EPTB patients (C) or healthy controls (D) from Adult and Child. Lines in the scatter plots represent median values. Data were compared using the MannWhitney U test. $* \mathrm{P}<0.05 ; * * \mathrm{P}<0.01 ; * * * \mathrm{P}<0.0001$. 

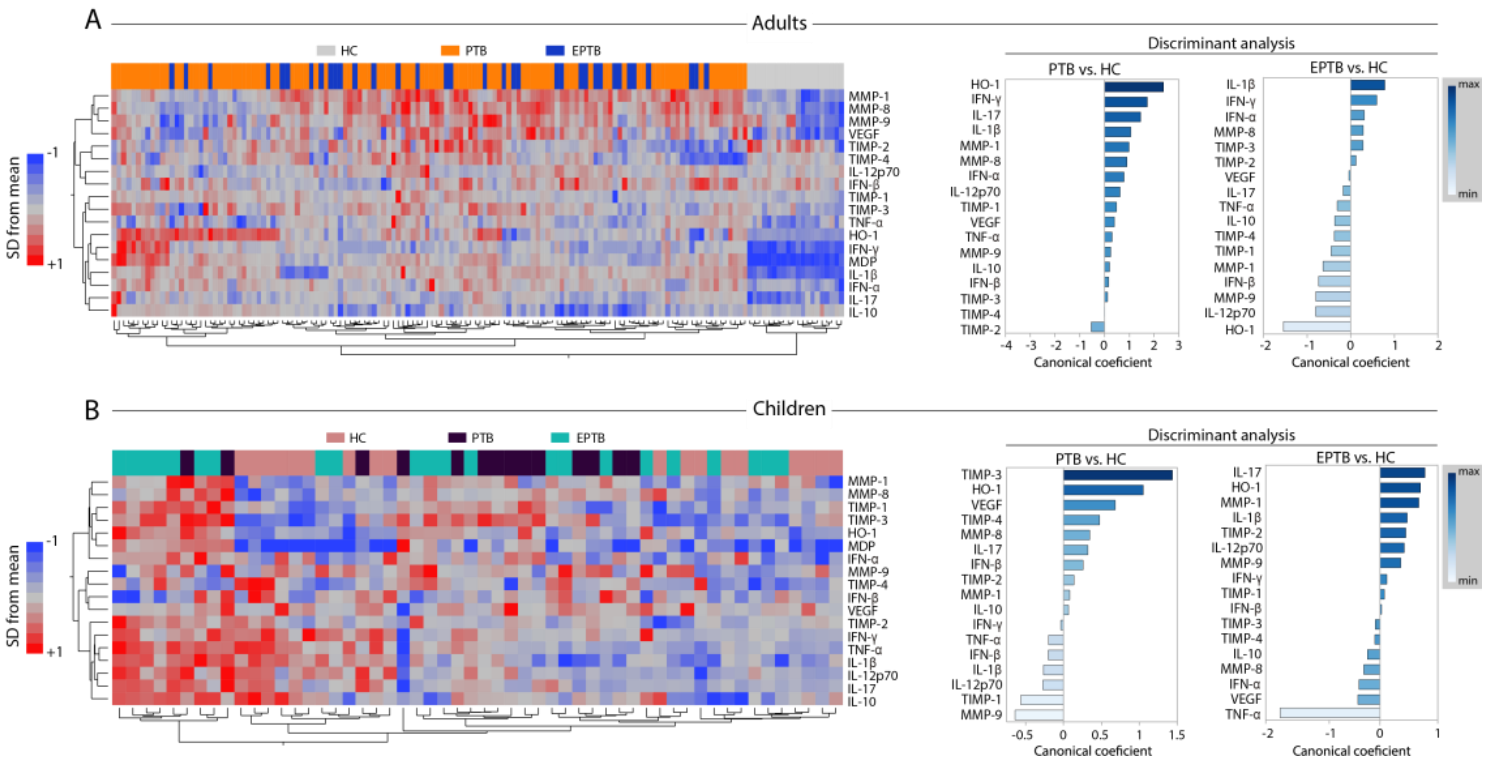

Figure 2. Plasma biomarkers driving the overall molecular degree of perturbation in pulmonary tuberculosis are distinct between Adult and Children patients. (A,B) Left panels: Unsupervised twoway hierarchical cluster analyses (Wards method with 100x bootstrap) using the MDP values for each individual markers measured in plasma from patients from both groups were employed to test if simultaneous assessment of such markers could group PTB or EPTB separately from healthy individuals. Dendrograms represent Euclidean distance. Right panels: A discriminant analysis model based on canonical correlation analyses was used to identify the markers which are driving the discrimination between the study groups. Number of patients per group: Adult HC: $n=20$, Adult PTB: $\mathrm{n}=97$, Adult EPTB: $\mathrm{n}=35$, Child HC: $\mathrm{n}=18$, Child PTB: $\mathrm{n}=14$, Child EPTB: $\mathrm{n}=22$. 
A

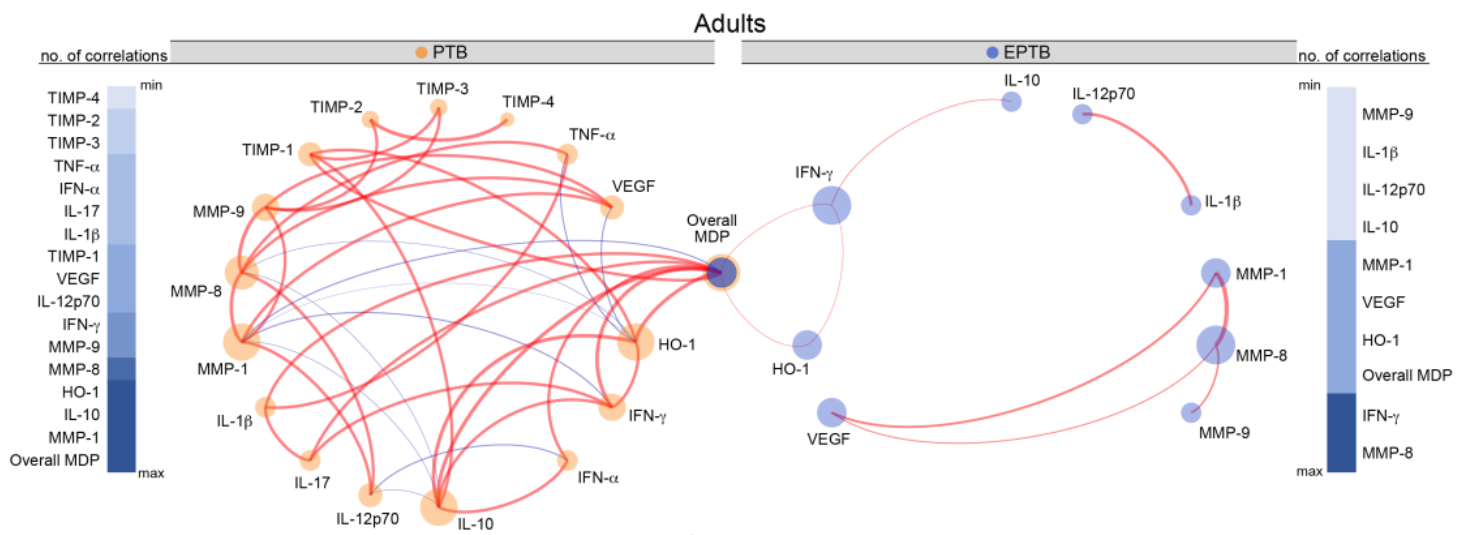

B

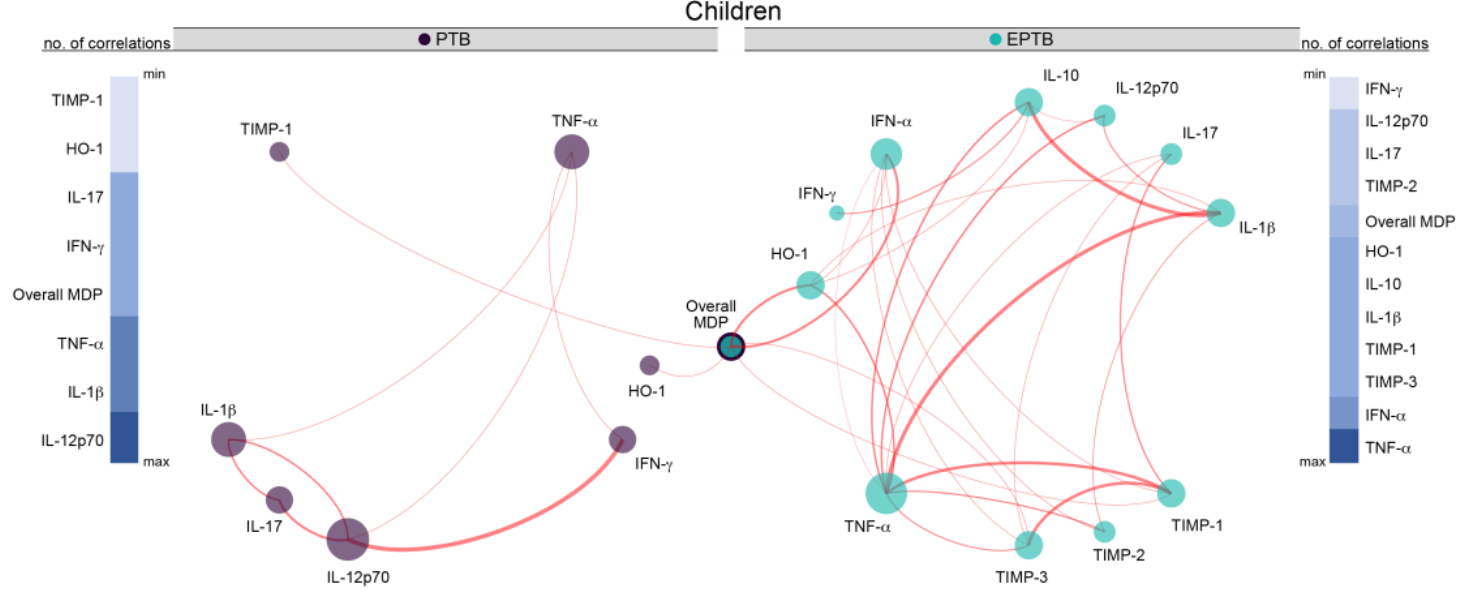

Figure 3: Network analysis of the MDP matrices in the study groups. (A,B) Spearman correlation matrices of the biomarker expression levels in each study group were built and Circos plots were used to illustrate the correlation networks. Each circle represents a different plasma parameter. The size of each circle is proportional to the number of significant correlations. P-values were adjusted for multiple measurements using Holm-Bonferroni's method and the connecting lines represent statistically significant correlations $(\mathrm{p}<0.00277)$. Red connecting lines represent positive correlations while blue lines infer negative correlations. Color intensity is proportional to the strength of correlation (rho value). Node analysis was used to illustrate the number of significant correlations per marker. Markers were grouped according to the number of connections from minimum to maximum numbers detected. 
B

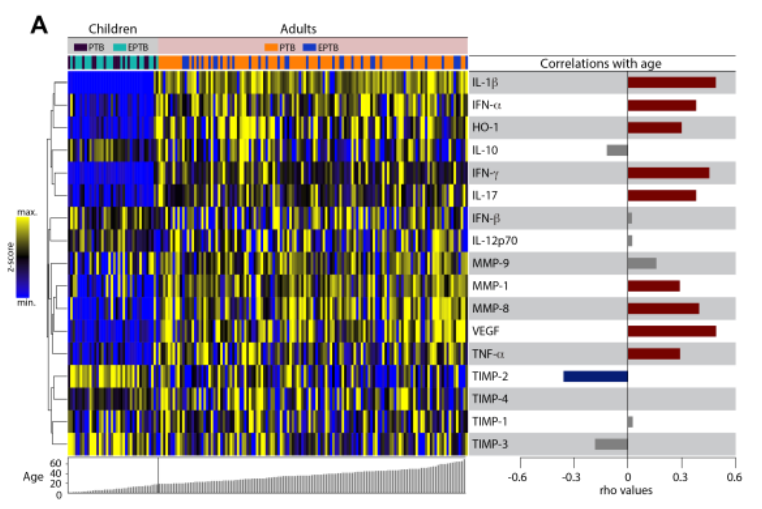

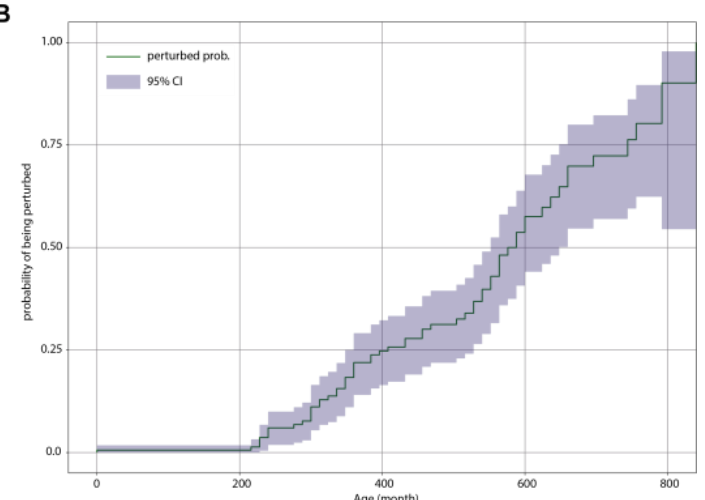

Figure 4: Associations between molecular degree inflammatory perturbation and age in TB patients. (A) Molecular degree of perturbation was assessed in samples from Adult and Child patients with tuberculosis. Data were z-score normalized. A hierarchical cluster analysis was employed to test whether the overall expression profile of the biomarkers could separate the study groups. Dendrograms represent Euclidean distance. Each individual was grouped based on age. The right panel shows Spearman correlation coefficient values of relationships between the indicated parameters and age. (B) Perturbed probability according to the age. Spearman correlation rank was compared used Steger Method. 
A

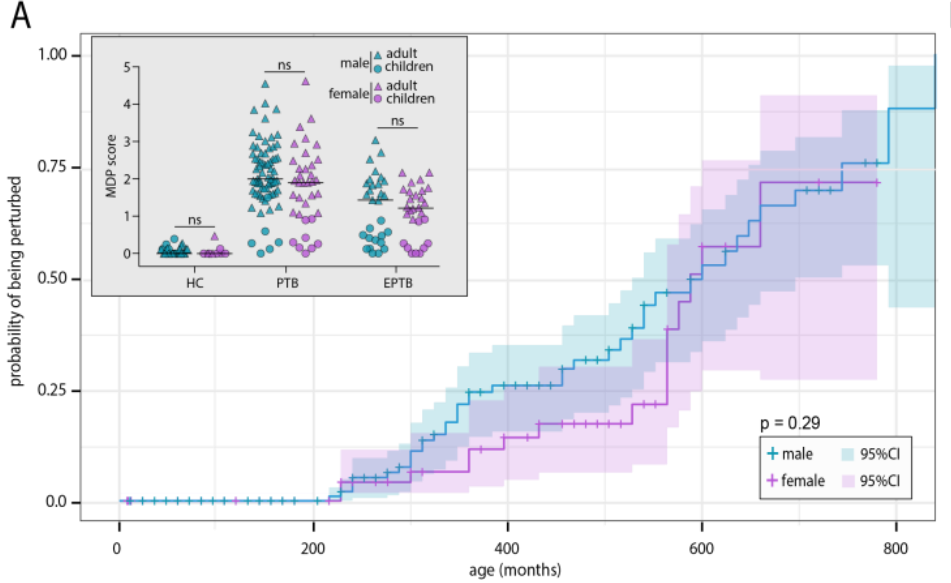

B

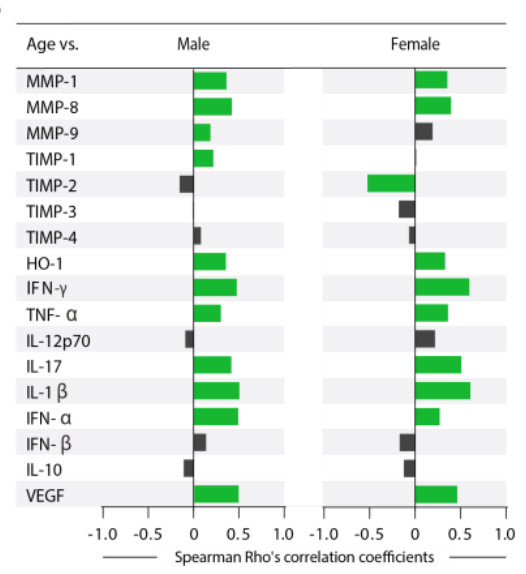

Figure 5: Molecular degree of perturbation is gender independent in patients with active

tuberculosis. (A) Perturbed probability according to the age. Spearman correlation rank was compared used Steger Method. (B) Spearman correlation analysis was used to test association between age and gender. Bars represent the Spearman rank (rho) values. Colored bars indicate statistically significant correlation $(\mathrm{p}<0.05)$ after adjustment for multiple measurement. 


\section{Supplemental Tables and Figures}

530

531

Table S1. Characteristics of adult participants

\begin{tabular}{lcccc}
\hline Characteristic & Healthy controls & PTB & EPTB & P-value \\
\hline $\mathrm{N}$ & 20 & 97 & 35 & \\
Age $-\mathrm{y}$ & $28.5(26-35)$ & $38(28-47)$ & $35(25-45)$ & 0.0452 \\
Male - no. (\%) & $17(85)$ & $64(65.9)$ & $16(45.7)$ & 0.0062
\end{tabular}

532 Data represent medians and interquartile ranges (age) and frequencies (male sex). The Kruskal-

533 Wallis test was used to compare distributions of age while the Pearson's chi-square test was

534 used to compare frequencies.

535 
bioRxiv preprint doi: https://doi.org/10.1101/2020.03.10.985697; this version posted March 11,2020 . The copyright holder for this preprint (which was not certified by peer review) is the author/funder, who has granted bioRxiv a license to display the preprint in perpetuity. It is made available under aCC-BY-NC-ND 4.0 International license.

Table S2. Characteristics of pediatric participants

\begin{tabular}{lcccc}
\hline Characteristic & Healthy controls & PTB & EPTB & P-value \\
\hline $\mathrm{N}$ & 18 & 14 & 22 & \\
Age $-\mathrm{y}$ & $10(3-12)$ & $6.5(1.7-12.5)$ & $7(3.7-13)$ & 0.6841 \\
Male - no. (\%) & $12(66.7)$ & $6(42.8)$ & $13(59.1)$ & 0.3928
\end{tabular}

537 Data represent medians and interquartile ranges (age) and frequencies (male sex). The Kruskal-

538 Wallis test was used to compare distributions of age while the Pearson's chi-square test was

539 used to compare frequencies.

540 
bioRxiv preprint doi: https://doi.org/10.1101/2020.03.10.985697; this version posted March 11,2020 . The copyright holder for this preprint (which was not certified by peer review) is the author/funder, who has granted bioRxiv a license to display the preprint in perpetuity. It is made available under aCC-BY-NC-ND 4.0 International license.

541 Table S3. Sex distribution in study population

\begin{tabular}{lcccc}
\hline Group & Sex & Adult & Children & P-value \\
\hline \multirow{2}{*}{ HC } & Male & 17 & 12 & 0.2603 \\
& Female & 3 & 6 & 0.1372 \\
\multirow{2}{*}{ PTB } & Male & 64 & 6 & \\
\multirow{2}{*}{ EPTB } & Female & 33 & 8 & 0.4173 \\
\hline
\end{tabular}

542 Data represent number of individuals in each group/category. The Fisher's exact test was used

543 to compare frequencies. 
bioRxiv preprint doi: https://doi.org/10.1101/2020.03.10.985697; this version posted March 11,2020 . The copyright holder for this preprint (which was not certified by peer review) is the author/funder, who has granted bioRxiv a license to display the preprint in perpetuity. It is made available under aCC-BY-NC-ND 4.0 International license.

Table S4. Distribution of the plasma concentrations of the mediators of inflammation in adult participants

\begin{tabular}{|c|c|c|c|c|c|}
\hline Parameter & Unit & Healthy controls & PTB & EPTB & P-value \\
\hline$N$ & & 20 & 97 & 35 & \\
\hline Heme Oxygenase-1 & $\mathrm{ng} / \mathrm{ml}$ & $\begin{array}{c}1.5 \\
(1.3-1.6)\end{array}$ & $\begin{array}{c}5.6 \\
(3.2-11.6)\end{array}$ & $\begin{array}{c}3.8 \\
(2.3-6.5)\end{array}$ & $<0.0001$ \\
\hline IFN- $\alpha$ & $\mathrm{pg} / \mathrm{mL}$ & $\begin{array}{c}3.2 \\
(1-4.3)\end{array}$ & $\begin{array}{c}11.6 \\
(7.9-17.3)\end{array}$ & $\begin{array}{c}8.3 \\
(4.8-12.1)\end{array}$ & $<0.0001$ \\
\hline IFN- $\beta$ & $\mathrm{pg} / \mathrm{mL}$ & $\begin{array}{c}4.4 \\
(2.2-5.5)\end{array}$ & $\begin{array}{c}2.9 \\
(1.3-7.1)\end{array}$ & $\begin{array}{c}3.4 \\
(1.5-5.9)\end{array}$ & 0.8231 \\
\hline IFN- $\nu$ & $\mathrm{pg} / \mathrm{mL}$ & $\begin{array}{c}20.6 \\
(15-19.5)\end{array}$ & $\begin{array}{c}33.9 \\
(29.8-35.3)\end{array}$ & $\begin{array}{c}29.8 \\
(27.8-36.0)\end{array}$ & $<0.0001$ \\
\hline IL-10 & $\mathrm{pg} / \mathrm{mL}$ & $\begin{array}{c}18.4 \\
(16.43-20.85)\end{array}$ & $\begin{array}{c}21.0 \\
(15.93-29.05)\end{array}$ & $\begin{array}{c}14.8 \\
(10.3-21.0)\end{array}$ & 0.0014 \\
\hline IL-12p70 & $\mathrm{pg} / \mathrm{mL}$ & $\begin{array}{c}2.3 \\
(1.6-3.3)\end{array}$ & $\begin{array}{c}2.2 \\
(0.9-5.3)\end{array}$ & $\begin{array}{c}2.9 \\
(1.5-6.9)\end{array}$ & 0.2078 \\
\hline IL-17 & $\mathrm{pg} / \mathrm{mL}$ & $\begin{array}{c}16.3 \\
(43.1-493.1)\end{array}$ & $\begin{array}{c}27.1 \\
(25.85-35.32)\end{array}$ & $\begin{array}{c}26.5 \\
(23.2-30.0)\end{array}$ & $<0.0001$ \\
\hline IL-1 $\beta$ & $\mathrm{pg} / \mathrm{mL}$ & $\begin{array}{c}2.8 \\
(2.5-3.4)\end{array}$ & $\begin{array}{c}15.9 \\
(12.9-20.1)\end{array}$ & $\begin{array}{c}13.1 \\
(11.7-15.9)\end{array}$ & $<0.0001$ \\
\hline MMP-1 & $\mathrm{ng} / \mathrm{mL}$ & $\begin{array}{c}0.07 \\
(0.04-0.32)\end{array}$ & $\begin{array}{c}3.4 \\
(1.9-5.3)\end{array}$ & $\begin{array}{c}2.9 \\
(2.2-4.5)\end{array}$ & $<0.0001$ \\
\hline MMP-8 & $\mathrm{ng} / \mathrm{mL}$ & $\begin{array}{c}13.9 \\
(5.1-32.5)\end{array}$ & $\begin{array}{c}122.4 \\
(54.1-202.3)\end{array}$ & $\begin{array}{c}88.4 \\
(75.6-141.9)\end{array}$ & $<0.0001$ \\
\hline MMP-9 & $\mathrm{ng} / \mathrm{mL}$ & $\begin{array}{c}133.7 \\
(53.7-331.5)\end{array}$ & $\begin{array}{c}384.2 \\
(255.0-510.8)\end{array}$ & $\begin{array}{c}271.6 \\
(214.7-329.6)\end{array}$ & $<0.0001$ \\
\hline TIMP-1 & $\mathrm{ng} / \mathrm{mL}$ & $\begin{array}{c}151.4 \\
(136.0-166.2)\end{array}$ & $\begin{array}{c}180.0 \\
(161.7-199.7)\end{array}$ & $\begin{array}{c}169.6 \\
(153.3-186.5)\end{array}$ & $<0.0001$ \\
\hline TIMP-2 & $\mathrm{ng} / \mathrm{mL}$ & $\begin{array}{c}232.9 \\
(219.2-255.9)\end{array}$ & $\begin{array}{c}218.9 \\
(199.4-238.7)\end{array}$ & $\begin{array}{c}223.0 \\
(208.3-234.1)\end{array}$ & 0.1001 \\
\hline TIMP-3 & $\mathrm{ng} / \mathrm{mL}$ & $\begin{array}{c}12.4 \\
(5.5-19.2)\end{array}$ & $\begin{array}{c}26.3 \\
(15.9-43.9)\end{array}$ & $\begin{array}{c}19.8 \\
(11.5-29.4)\end{array}$ & $<0.0001$ \\
\hline TIMP-4 & $\mathrm{ng} / \mathrm{mL}$ & $\begin{array}{c}9.6 \\
(8.4-10.8)\end{array}$ & $\begin{array}{c}10.3 \\
(7.7-12.4)\end{array}$ & $\begin{array}{c}8.8 \\
(7.5-10.9)\end{array}$ & 0.2174 \\
\hline TNF- $\alpha$ & $\mathrm{pg} / \mathrm{mL}$ & $\begin{array}{c}12.5 \\
(10.2-17.5)\end{array}$ & $\begin{array}{c}21.3 \\
(14.9-27.4)\end{array}$ & $\begin{array}{c}18.9 \\
(15.5-23.9)\end{array}$ & 0.0006 \\
\hline VEGF & $\mathrm{pg} / \mathrm{mL}$ & $\begin{array}{c}26.0 \\
(18.4-47.8)\end{array}$ & $\begin{array}{c}100.8 \\
(57.9-165.5)\end{array}$ & $\begin{array}{c}71.5 \\
(43.0-113.3)\end{array}$ & $<0.0001$ \\
\hline
\end{tabular}

547 Data represent medians and interquartile ranges. The Kruskal-Wallis test was used to compare 548 the distributions of the plasma mediators between the study groups. P-values in bold font are 549 statistically significant. 
Table S5. Distribution of the plasma concentrations of the mediators of inflammation in pediatric participants

\begin{tabular}{|c|c|c|c|c|c|}
\hline Parameter & Unit & Healthy controls & PTB & EPTB & P-value \\
\hline$N$ & & 18 & 14 & 22 & \\
\hline Heme Oxygenase-1 & $\mathrm{ng} / \mathrm{ml}$ & $\begin{array}{c}0.5 \\
(0.4-0.6)\end{array}$ & $\begin{array}{c}0.6 \\
(0.5-1.0)\end{array}$ & $\begin{array}{c}0.9 \\
(0.6-1.1)\end{array}$ & 0.0005 \\
\hline IFN- $\alpha$ & $\mathrm{pg} / \mathrm{mL}$ & $\begin{array}{c}209.7 \\
(11.2-380.8)\end{array}$ & $\begin{array}{c}70.0 \\
(10.9-87.9)\end{array}$ & $\begin{array}{c}135.6 \\
(21.4-735.8)\end{array}$ & 0.1903 \\
\hline IFN- $\beta$ & $\mathrm{pg} / \mathrm{mL}$ & $\begin{array}{c}347.9 \\
(233.4-511.0)\end{array}$ & $\begin{array}{c}312 \\
(261.1-466.0)\end{array}$ & $\begin{array}{c}286.8 \\
(200.0-427.7)\end{array}$ & 0.5615 \\
\hline IFN- $\nu$ & $\mathrm{pg} / \mathrm{mL}$ & $\begin{array}{c}244.7 \\
(111.4-812.5)\end{array}$ & $\begin{array}{c}377.4 \\
(252.4-720.5)\end{array}$ & $\begin{array}{c}325.4 \\
(178.3-892.6)\end{array}$ & 0.4723 \\
\hline IL-10 & $\mathrm{pg} / \mathrm{mL}$ & $\begin{array}{c}15.6 \\
(0.4-80.4)\end{array}$ & $\begin{array}{c}11.3 \\
(10.0-13.1)\end{array}$ & $\begin{array}{c}5.3 \\
(0.5-97.0)\end{array}$ & 0.8904 \\
\hline IL-12p70 & $\mathrm{pg} / \mathrm{mL}$ & $\begin{array}{c}10.6 \\
(5.8-69.4)\end{array}$ & $\begin{array}{c}16.8 \\
(7.9-55.9)\end{array}$ & $\begin{array}{c}13.6 \\
(7.1-48.8)\end{array}$ & 0.8443 \\
\hline IL-17 & $\mathrm{pg} / \mathrm{mL}$ & $\begin{array}{c}7.8 \\
(5.8-25.6)\end{array}$ & $\begin{array}{c}9.6 \\
(5.5-21.3)\end{array}$ & $\begin{array}{c}9.4 \\
(7.1-26.7)\end{array}$ & 0.7314 \\
\hline IL-1 $\beta$ & $\mathrm{pg} / \mathrm{mL}$ & $\begin{array}{c}85.9 \\
(13.9-174.2)\end{array}$ & $\begin{array}{c}28.6 \\
(2.3-87.4)\end{array}$ & $\begin{array}{c}42.3 \\
(8.2-478.9)\end{array}$ & 0.5183 \\
\hline MMP-1 & $\mathrm{ng} / \mathrm{mL}$ & $\begin{array}{c}0.3 \\
(0.2-0.4)\end{array}$ & $\begin{array}{c}0.5 \\
(0.4-0.6)\end{array}$ & $\begin{array}{c}0.5 \\
(0.3-0.7)\end{array}$ & 0.0028 \\
\hline MMP-8 & $\mathrm{ng} / \mathrm{mL}$ & $\begin{array}{c}6.4 \\
(4.0-9.0)\end{array}$ & $\begin{array}{c}8.2 \\
(7.8-11.5)\end{array}$ & $\begin{array}{c}8.5 \\
(6.1-11.3)\end{array}$ & 0.0367 \\
\hline MMP-9 & $\mathrm{ng} / \mathrm{mL}$ & $\begin{array}{c}17.8 \\
(13.5-25.5)\end{array}$ & $\begin{array}{c}23.1 \\
(17.6-25.9)\end{array}$ & $\begin{array}{c}21.1 \\
(14.6-35.2)\end{array}$ & 0.4514 \\
\hline TIMP-1 & $\mathrm{ng} / \mathrm{mL}$ & $\begin{array}{c}2.3 \\
(1.7-3.0)\end{array}$ & $\begin{array}{c}4.0 \\
(2.6-5.3)\end{array}$ & $\begin{array}{c}3.4 \\
(1.9-5.1)\end{array}$ & 0.0263 \\
\hline TIMP-2 & $\mathrm{ng} / \mathrm{mL}$ & $\begin{array}{c}1.4 \\
(1.1-1.8)\end{array}$ & $\begin{array}{c}1.7 \\
(1.4-2.1)\end{array}$ & $\begin{array}{c}1.6 \\
(1.5-2.4)\end{array}$ & 0.1981 \\
\hline TIMP-3 & $\mathrm{ng} / \mathrm{mL}$ & $\begin{array}{c}0.2 \\
(0.2-0.3)\end{array}$ & $\begin{array}{c}0.7 \\
(0.5-0.8)\end{array}$ & $\begin{array}{c}0.4 \\
(0.3-0.5)\end{array}$ & $<0.0001$ \\
\hline TIMP-4 & $\mathrm{ng} / \mathrm{mL}$ & $\begin{array}{c}0.02 \\
(0.02-0.03)\end{array}$ & $\begin{array}{c}0.02 \\
(0.02-0.03)\end{array}$ & $\begin{array}{c}0.02 \\
(0.01-0.04)\end{array}$ & 0.8515 \\
\hline TNF- $\alpha$ & $\mathrm{pg} / \mathrm{mL}$ & $\begin{array}{c}162.7 \\
(37.1-1691.1)\end{array}$ & $\begin{array}{c}87.6 \\
(53.4-203.4)\end{array}$ & $\begin{array}{c}197.1 \\
(88.1-735.0)\end{array}$ & 0.3645 \\
\hline VEGF & $\mathrm{pg} / \mathrm{mL}$ & $\begin{array}{c}71.0 \\
(56.0-136.6)\end{array}$ & $\begin{array}{c}111.9 \\
(78.6-127.8)\end{array}$ & $\begin{array}{c}86.1 \\
(56.0-105.8)\end{array}$ & 0.3415 \\
\hline
\end{tabular}

553 Data represent medians and interquartile ranges. The Kruskal-Wallis test was used to compare 554 the distributions of the plasma mediators between the study groups. P-values in bold font are 555 statistically significant. 

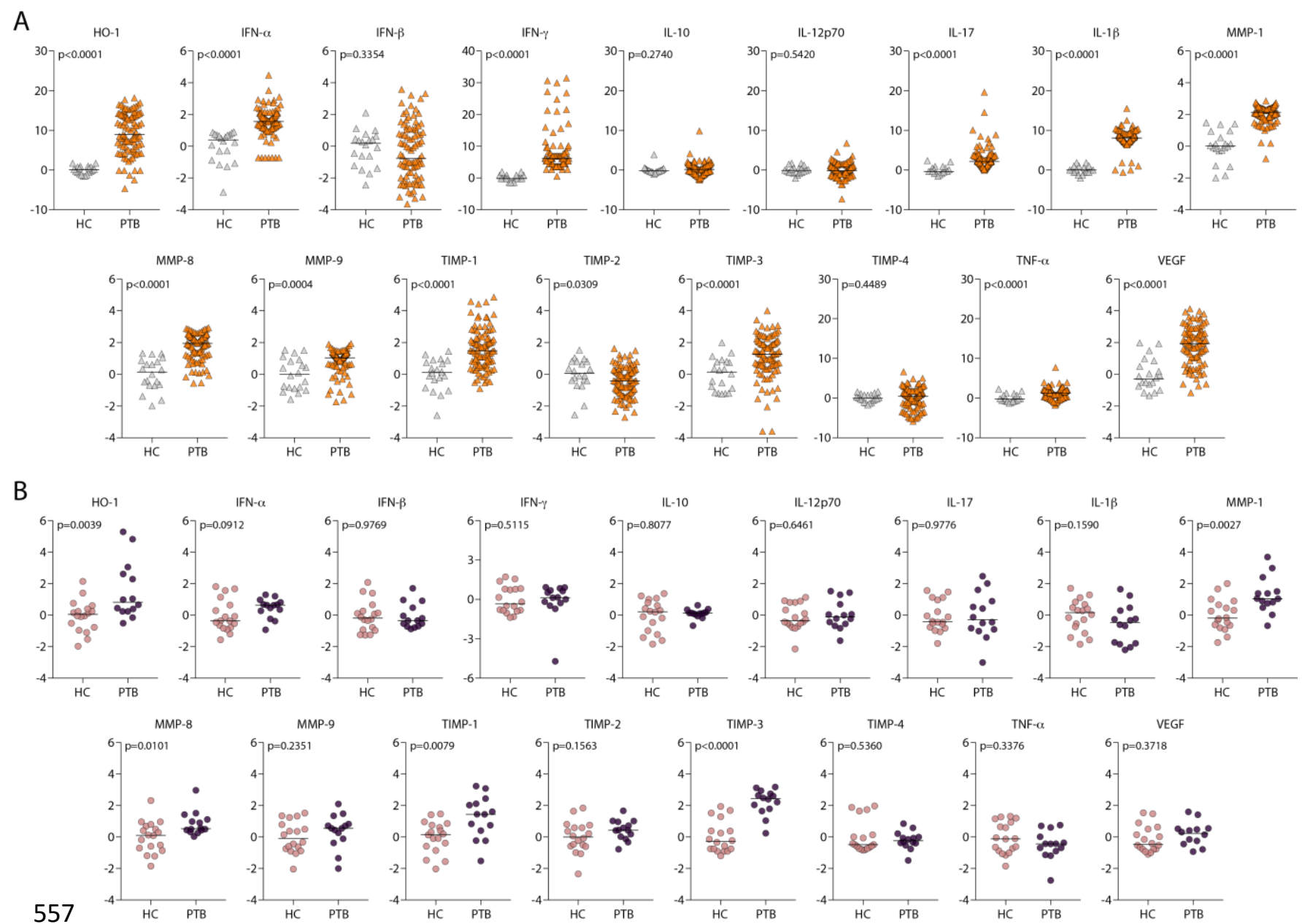

Figure S1. Differences in the inflammatory perturbation of each plasma biomarker between active PTB and healthy control groups in either adults or children. (A,B) Scatterplots of molecular degree of perturbation (MDP) of indicated biomarkers of TB patients stratified per the group (Adult HC, $n=20$ and PTB, $n=97$; Children HC, $n=18$ and PTB, $n=14$ ). MDP scores are shown in $Y$-axis from all the plots. Lines represent median values. Data were compared using the Mann-Whitney $U$ test. 
A
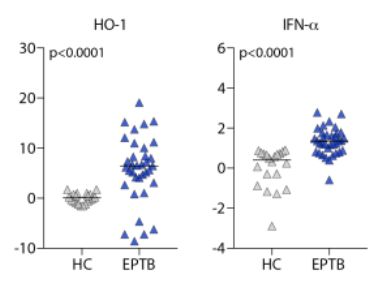

MMP-8
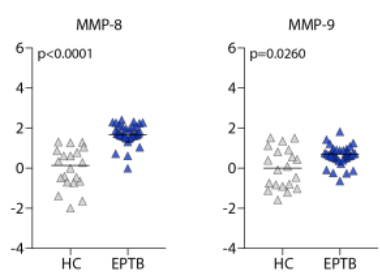
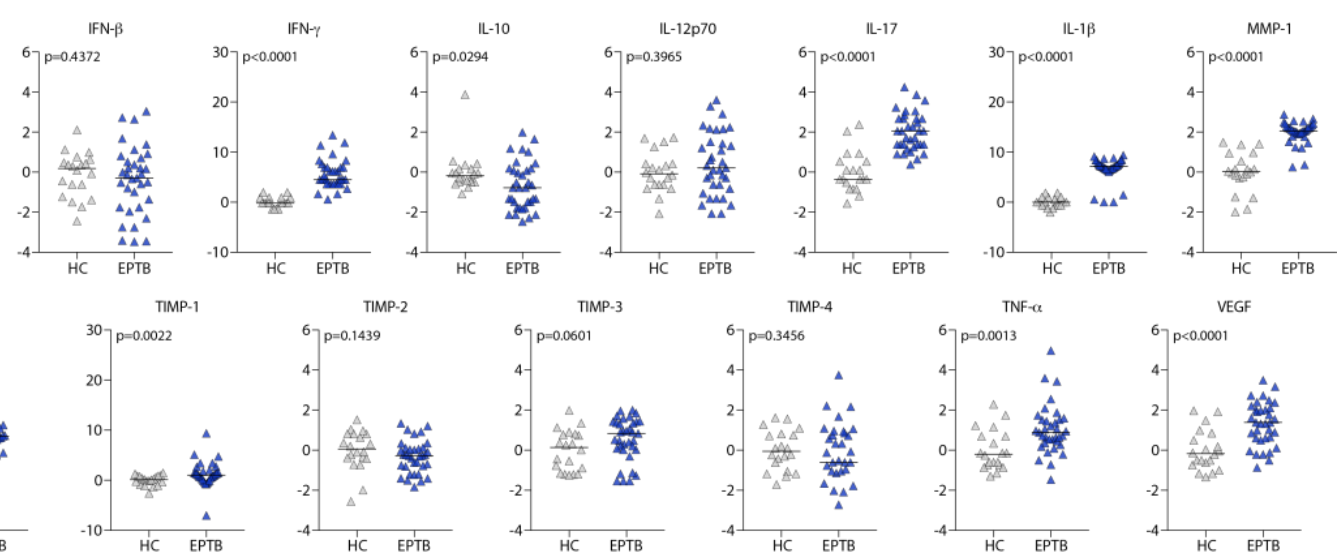

VEGF

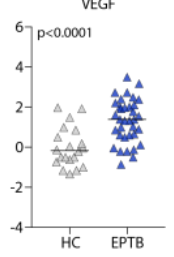

B
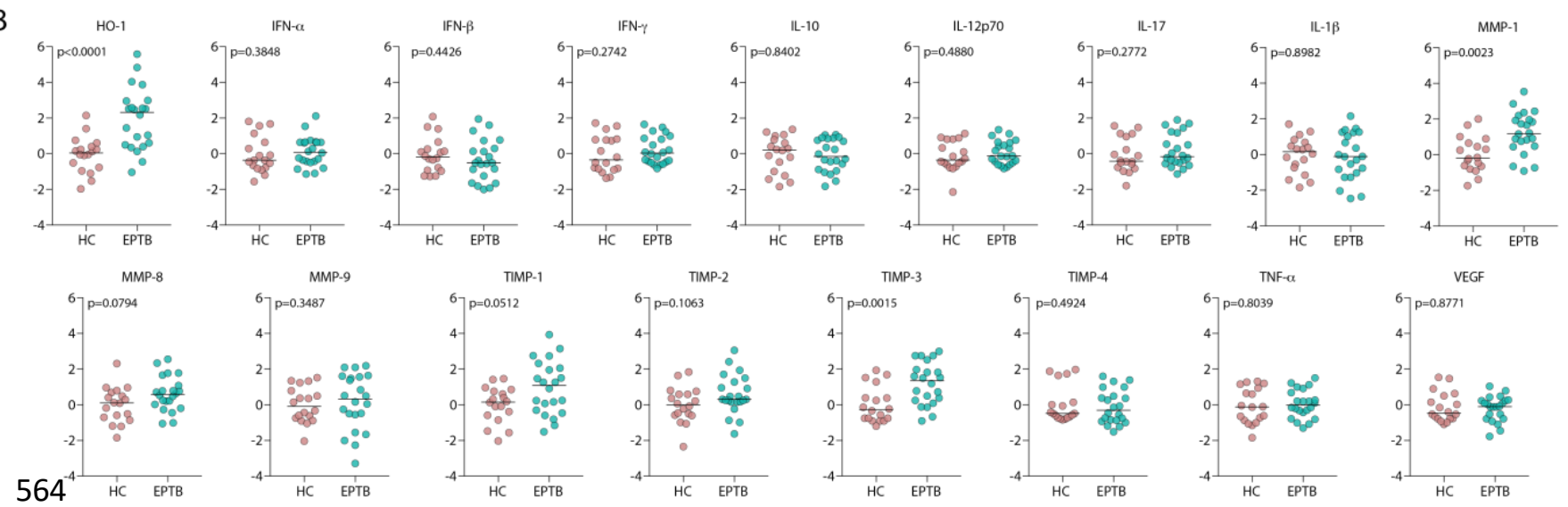

565 Figure S2. Differences in the inflammatory perturbation between adult or children with extrapulmonary

566 tuberculosis compared to healthy controls. (A,B) Scatterplots of molecular degree of perturbation (MDP)

567 of indicated biomarkers of TB patients stratified per the group (Adult HC, $n=20$ and EPTB, $n=35$; Children

568 HC, $n=18$ and EPTB, $n=22$ ). MDP scores are shown in $Y$-axis from all the plots. Lines represent median 569 values. Data were compared using the Mann-Whitney $U$ test. 
bioRxiv preprint doi: https://doi.org/10.1101/2020.03.10.985697; this version posted March 11,2020 . The copyright holder for this preprint (which was not certified by peer review) is the author/funder, who has granted bioRxiv a license to display the preprint in perpetuity. It is made available under aCC-BY-NC-ND 4.0 International license.
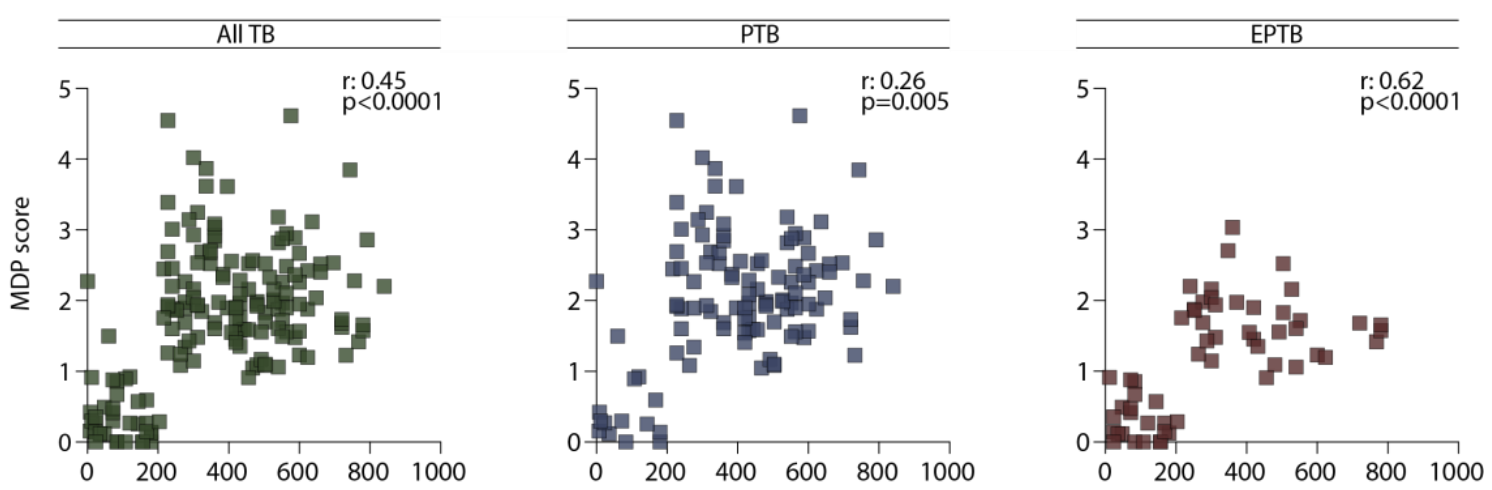

571 Age (month)

\section{Figure S3. Association between Age and MDP score in patients with tuberculosis}

573 Correlation between Age and MDP score was assessed using the Spearman rank test in the 574 different study groups. 